V. M. Petechuk, Yu. V. Petechuk

\title{
PROPERTIES OF THE COMMUTATORS OF SOME ELEMENTS OF LINEAR GROUPS OVER DIVISIONS RINGS
}

\begin{abstract}
V. M. Petechuk, Yu. V. Petechuk. Properties of the commutators of some elements of linear groups over divisions rings, Mat. Stud. 54 (2020), 15-22.

Inclusions resulting from the commutativity of elements and their commutators with transvections in the language of residual and fixed submodules are found.

The residual and fixed submodules of an element $\sigma$ of the complete linear group are defined as the image and the kernel of the element $\sigma-1$ and are denoted by $R(\sigma)$ and $P(\sigma)$, respectively.

It is shown that for an arbitrary element $g$ of a complete linear group over a division ring whose characteristic is different from 2 and the transvection $\tau$ from the commutativity of the commutator $[g, \tau]$ with $g$ is followed by the inclusion of $R([g, \tau]) \subseteq P(\tau) \cap P(g)$. It is proved that the same inclusions occur over an arbitrary division ring if $g$ is a unipotent element, $\operatorname{dim}(R(\tau)+R(g)) \leq 2$ and the commutator $[g, \tau]$ commutes with $\tau$ or if $g$ is a unipotent commutator of some element of the complete linear group and transvection $\tau$.
\end{abstract}

\section{Dedicated to the bright memory of Professor B. V. Zabavsky}

Introduction. This article studies the conditions of commutativity of commutators with group elements $G L(n, R), n \geq 2$.

It is shown that if $R$ is a division ring and char $R \neq 2$, then the inclusion of $R([g, \tau]) \subseteq$ $P(\tau) \cap P(g)$ follows from the commutativity of the elements $g$ and $[g, \tau]$. If $R$ is a division ring and char $R=2$, then this statement does not hold.

The paper proves that the inclusion of $R([g, \tau]) \subseteq P(\tau) \cap P(g)$ also occurs if $R$ is a division ring, $g$ is a unipotent element, $\tau$ is a transvection of the group $G L(n, R), \operatorname{dim}(R(\tau)+R(g)) \leq$ 2 and $[g, \tau]$ commutes with $\tau$ or if $g$ is a unipotent commutator of some element of the group $G L(n, R), n \geq 2$ and transvection $\tau$.

The main results are presented in Theorems 1-3.

Let $V$ be the left free module of finite rank $n \geq 2$ over the ring $R$. The group of linear automorphisms of module $V$ will be denoted by $G L(n, V)$ and will be called the complete linear group of module $V$. As is known in the fixed base of module $V$, this group is identified with the complete linear group $G L(n, R)$ above the ring $R$. Conditions for the commutativity of transvections with elements of linear groups over division ring are considered in the language of residual and fixed submodules. The residual and fixed submodules of an element $\sigma$ of the complete linear group $G L(n, V)$ of the module $V$ over the ring $R$ are defined as the image and the kernel of the element $\sigma-1$ and are denoted by $R(\sigma)$ and $P(\sigma)$, respectively.

Obviously that

$R\left(\sigma^{-1}\right)=R(\sigma), P\left(\sigma^{-1}\right)=P(\sigma)$ i $R\left(g \sigma g^{-1}\right)=g R(\sigma), P\left(g \sigma g^{-1}\right)=g P(\sigma)$,

2010 Mathematics Subject Classification: 12E15, $15 \mathrm{~B} 33$.

Keywords: division ring; linear groups; residual and fixed modules; transvections; unipotent elements; commutators; commutativity.

doi:10.30970/ms.54.1.15-22

(C) V. M. Petechuk, Yu. V. Petechuk, 2020 
where $g \in G L(n, V)$.

It is easy to see that $R\left(\sigma_{1} \sigma_{2}\right) \subseteq R\left(\sigma_{1}\right)+R\left(\sigma_{2}\right)$ and

$$
R\left(\left[\sigma_{1}, \sigma_{2}\right]\right)=R\left(\sigma_{1} \sigma_{2} \sigma_{1}^{-1} \sigma_{2}^{-1}\right) \subseteq \sigma_{1} R\left(\sigma_{1}\right)+R\left(\sigma_{2}\right) \subseteq R\left(\sigma_{1}\right)+R\left(\sigma_{2}\right) .
$$

Note that the submodules of the module $V$ that contain $R(\sigma)$ or are contained in $P(\sigma)$ are invariant with respect to $\sigma$.

The inclusion of $R\left(\sigma_{1}\right) \subseteq P\left(\sigma_{2}\right)$ is equivalent to the equality $\left(\sigma_{2}-1\right)\left(\sigma_{1}-1\right)=0$ or the equality $\sigma_{1} \sigma_{2}=\sigma_{1}+\sigma_{2}-1$ for any elements $\sigma_{1}$ and $\sigma_{2}$ of the group $G L(n, V), n \geq 2$ of the left module $V$ over the ring $R$.

Therefore, from the system

$$
\left\{\begin{array}{l}
R\left(\sigma_{1}\right) \subseteq P\left(\sigma_{2}\right) \\
R\left(\sigma_{2}\right) \subseteq P\left(\sigma_{1}\right)
\end{array}\right.
$$

the commutativity $\sigma_{1}$ and $\sigma_{2}$ follows.

It is clear that the converse is not always true.

However, if $\sigma_{1}$ and $\sigma_{2}$ are commutators and one of the inclusions of the system (1) takes place, then the second inclusion of the system (1) also takes place. We will use this property in the future without further explanation.

An element $\sigma$ of the group $G L(n, V)$ is called unipotent if $\sigma-1$ is a nilpotent element, i.e. if there exists a natural number $k$ such that $(\sigma-1)^{k}=0$.

The least $k>0$ such that $(\sigma-1)^{k}=0$ is called the level of the nilpotent element $\sigma-1$ (respectively unipotent element $\sigma$ ).

In what follows, we assume that $R$ is an arbitrary division ring.

The element $\tau$ of the group $G L(n, V)$ will be called transvection if $R(\tau) \subseteq P(\tau)$ and $\operatorname{dim} R(\tau)=1$.

We assume that the transvection $\tau$ is a class of all transvections whose residual and fixed modules coincide with the residual and fixed modules $\tau$, respectively.

Obviously, element 1 is a unipotent element of level 1 and transvection is a unipotent element of level 2.

If we fix the base $e_{1}, \ldots, e_{n}$ of the module $V$ so that $R(\tau)=\left\langle e_{i}\right\rangle, P(\tau)=\left\langle e_{1}, \ldots, e_{j-1}\right.$, $\left.e_{j+1}, \ldots, e_{n}\right\rangle, 1 \leq i \neq j \leq n$, then in this base the transvection $\tau$ has the form $t_{i j}(r)=1+r e_{i j}$, where $0 \neq r \in R, e_{i j}$ is a standard matrix unit in which 1 is in place $(i, j)$, and in other places there are zeros.

The matrices $t_{i j}(r)$ are called elementary transvections. It is clear that the class of transvections $\tau$ in the above-mentioned base coincides with the group of elementary transvections $t_{i j}(r)$.

In [1] it is proved that if the transvection $\sigma_{1}$ commutes with the unipotent element $\sigma_{2}$, then system (1) takes place. An overview of issues and literature related to linear groups over divisions ring can be found in [2]-[5].

Lemma 1. Let $R$ be a division ring, $\tau$ be a transvection, and $g$ be an arbitrary element of the group $G L(n, V), n \geq 2, \tau^{g}=g \tau g^{-1},[g, \tau]=g \tau g^{-1} \tau^{-1}=\tau^{g} \tau^{-1}, g^{-1} R(\tau) \subseteq P(\tau)$. Then $[g, \tau]$ is a unipotent element of level $\leq 3$ and $R(\tau) \subseteq P([g, \tau])$.

Proof. Let $a=\tau^{-1}-1, b=\tau^{g}-1$. Then $a^{2}=b^{2}=0$. Because $R\left(\tau^{-1}\right)=R(\tau) \in g P(\tau)=$ $P\left(\tau^{g}\right)$, then $b a=0$. According to the designation

$$
[g, \tau]=\tau^{g} \tau^{-1}=(b+1)(a+1)=a+b+1, \quad([g, \tau]-1)\left(\tau^{-1}-1\right)=(a+b) a=0 .
$$


So $R(\tau)=R\left(\tau^{-1}\right) \subseteq P([g, \tau])$. In addition, the equalities $([g, \tau]-1)^{2}=(a+b)^{2}=a b$ and $([g, \tau]-1)^{3}=(a+b) a b=0$ hold.

So, $[\sigma, \tau]$ is unipotent element of level $\leq 3$.

Lemma 2. Let $R$ be a division ring, $\tau$ be a transvection, and $g$ be an arbitrary element of the group $G L(n, V), n \geq 2, \tau^{g}=g \tau g^{-1},[g, \tau]=g \tau g^{-1} \tau^{-1}=\tau^{g} \tau^{-1}, g R(\tau) \subseteq P(\tau)$. Then $[g, \tau]$ is a unipotent element of level $\leq 3$ and $R([g, \tau]) \subseteq P(\tau)$.

Proof. Let $a=\tau^{-1}-1, b=\tau^{g}-1$. Then $a^{2}=b^{2}=0$. Since $R\left(\tau^{g}\right)=g R(\tau) \subseteq$ $P(\tau)=P\left(\tau^{-1}\right)$, we have $a b=0,[g, \tau]=\tau^{g} \tau^{-1}=(b+1)(a+1)=b a+a+b+1$, $\left(\tau^{-1}-1\right)([g, \tau]-1)=a(b a+a+b)=0$. That means that $R([g, \tau]) \subseteq P\left(\tau^{-1}\right)=P(\tau)$.

In addition $([g, \tau]-1)^{2}=(b a+a+b)^{2}=b a$ and $([g, \tau]-1)^{3}=(b a+a+b) b a=0$

The next lemma follows from Lemma 1 and Lemma 2.

Lemma 3. Let $R$ be a division ring, $\tau$ be a transvection, and $g$ be an arbitrary element of the group $G L(n, V), n \geq 2, g^{ \pm 1} R(\tau) \subseteq P(\tau)$. Then the commutator $[g, \tau]$ is a unipotent element of level $\leq 2$ which commutes with $\tau$.

Proof. Let, as in Lemmas 1 and $2 a=\tau^{-1}-1, b=\tau^{g}-1$. Then $a^{2}=b^{2}=a b=b a=0$, $([g, \tau]-1)^{2}=(a+b)^{2}=0$ and there is a system of inclusions

$$
\left\{\begin{array}{l}
R(\tau) \subseteq P([g, \tau]) \\
R([g, \tau]) \subseteq P(\tau)
\end{array},\right.
$$

from which the commutativity of the commutator $[g, \tau]$ and transvection $\tau$ follows.

The commutator $[g, \tau]$ is a unipotent element of level $\leq 2$.

Lemma 4. Let $R$ be a division ring, $\tau$ be a transvection, and $g$ be an arbitrary element of the group $G L(n, V), n \geq 2$, submodules $g^{ \pm 1} R(\tau)$ do not belong to $P(\tau)$. Then the commutator $[g, \tau]$ is not a unipotent element, $\operatorname{dim} R([g, \tau])=2, R([g, \tau])=R(\tau) \oplus g R(\tau)$, $V=R([g, \tau]) \oplus P([g, \tau]))$.

Proof. Sinse $\tau$ is a transvection, then $\operatorname{dim} R(\tau)=1, \operatorname{dim} P(\tau)=n-1$. Choose the base $e_{1}, \ldots, e_{n}$ of the module $V$ such that $R(\tau)=\left\langle e_{1}\right\rangle, P(\tau)=\left\langle e_{1}, \ldots, e_{n-1}\right\rangle$.

Since the submodule $g R(\tau)$ does not belong to $P(\tau)$, we have $V=\left\langle g e_{1}\right\rangle \oplus P(\tau)$. Therefore, we can assume that $e_{n}=g e_{1}$. So $g R(\tau)=\left\langle e_{n}\right\rangle, e_{1}=g^{-1} e_{n}$.

Similarly, $g^{-1} R(\tau)$ does not belong $P(\tau)$. Then $V=\left\langle g^{-1} e_{1}\right\rangle \oplus P(\tau)$. This means that there exists $0 \neq x \in R$, such that $g^{-1} e_{1}-x g e_{1} \in P(\tau)$.

Then $(\tau-1)\left(g^{-1} e_{1}-x e_{n}\right)=0$.

Because $R(\tau)=(\tau-1) V \subseteq P(\tau)$, then $(\tau-1) e_{n}=r e_{1}$ for some $0 \neq r \in R$. So $\tau g^{-1} e_{1}=g^{-1} e_{1}+x r e_{1}$.

Note that $\left(1-\tau^{-1}\right) e_{n}=\tau^{-1}(\tau-1) e_{n}=\tau^{-1} r e_{1}=r e_{1}$ and $\tau^{-1} e_{n}=e_{n}-r e_{1}$.

From the obtained equations it follows that $[g, \tau] e_{1}=g \tau g^{-1} \tau^{-1} e_{1}=g\left(\tau g^{-1}\right) e_{1}=$ $g\left(g^{-1} e_{1}+x r e_{1}\right)=e_{1}+x r e_{n}$.

Similarly, it is proved that $[g, \tau] e_{n}=g \tau g^{-1} \tau^{-1} e_{n}=g \tau g^{-1}\left(e_{n}-r e_{1}\right)=g\left(\tau g^{-1} e_{n}-\right.$ $\left.-r\left(g^{-1} e_{1}+x r e_{1}\right)\right)=g e_{1}-r e_{1}-$ rxre $_{n}=e_{n}-r e_{1}-$ rxre $_{n}$. 
Thus it is proved that the submodule $\left\langle e_{1}, e_{n}\right\rangle$ is invariant with respect to the commutator $[g, \tau]$. The element $[g, \tau]-1$ on the submodule $\left\langle e_{1}, e_{n}\right\rangle$ is given by the inverse matrix

$$
\left(\begin{array}{cc}
0 & -r \\
x r & -r x r
\end{array}\right) \in G L(2, R)
$$

Then $[g, \tau]-1$ is not a nilpotent element and, as a consequence, the commutator $[g, \tau]$ is not a unipotent element.

Because $\operatorname{dim} R([g, \tau]) \leq 2$ and $\left\langle e_{1}, e_{n}\right\rangle \subseteq R([g, \tau])$, we have $R([g, \tau])=\left\langle e_{1}, e_{n}\right\rangle=$ $R(\tau) \oplus g R(\tau), \operatorname{dim} R([g, \tau])=2$. From the equality

$$
([g, \tau]-1) R([g, \tau]) \cap P([g, \tau])=0
$$

and the reversibility of the element $[g, \tau]-1$ on $R([g, \tau])$ it follows, that

$$
R([g, \tau]) \cap P([g, \tau])=0 .
$$

So $V=R([g, \tau]) \oplus P([g, \tau])$.

Theorem 1. Let $R$ be a division ring, char $R \neq 2, g \in G L(n, V), n \geq 2, \tau$ be a transvection such that the commutator $[g, \tau]$ commutes with $g$. Then $[g, \tau]$ is a unipotent element of level $\leq 2$, which commutes with $\tau$ and

$$
R([g, \tau]) \subseteq P(\tau) \cap P(g), R(\tau)+R(g) \subseteq P([g, \tau]) .
$$

Proof. Let us show that $g^{ \pm 1} R(\tau) \subseteq P(\tau)$. In this case, Theorem 1 will follow from Lemma 3.

Let $R(\tau)=\langle v\rangle \subseteq P(\tau)$ for some $0 \neq v \in V$. Then $(\tau-1) v=0$,

$$
(\tau-1) g v=\alpha v,(\tau-1) g^{-1} v=\beta v
$$

where $\alpha, \beta$ belong to $R$. We get that

$$
\left(\tau^{-1}-1\right) g v=-\alpha v,\left(\tau^{-1}-1\right) g^{-1} v=-\beta v .
$$

Since the commutator $[g, \tau]$ commutes with $g$, the elements $\tau g^{-1} \tau^{-1}$ and $\tau^{-1} g^{-1} \tau$ also commute with $g$. Direct verification establishes that

$$
\begin{aligned}
& \tau g^{-1} \tau^{-1} g v=\tau g^{-1}(g v-\alpha v)=v-\alpha\left(g^{-1} v+\beta v\right), \\
& g \tau g^{-1} \tau^{-1} v=g \tau g^{-1} v=g\left(g^{-1} v+\beta v\right)=v+\beta g v .
\end{aligned}
$$

Since the left parts of the equations are equal, we have

$$
\alpha g^{-1} v+\beta g v=-\alpha \beta v .
$$

Similarly

$$
\begin{gathered}
\tau^{-1} g^{-1} \tau g v=\tau^{-1} g^{-1}(g v+\alpha v)=v+\alpha \tau^{-1} g^{-1} v=v+\alpha\left(g^{-1} v-\beta v\right), \\
g \tau^{-1} g^{-1} \tau v=g \tau^{-1} g^{-1} v=g\left(g^{-1} v-\beta v\right)=v-\beta g v
\end{gathered}
$$

Then

$$
\alpha g^{-1} v+\beta g v=\alpha \beta v
$$


Thus it is proved that $2 \alpha \beta=0$. Since char $R \neq 2, \alpha \beta=0$. This means that

$$
\alpha g^{-1} v+\beta g v=0, \alpha^{2} g^{-1} v=\beta^{2} g v=0
$$

and as a result, $\alpha=\beta=0, g^{ \pm 1} R(\tau) \subseteq P(\tau)$.

Note that the inclusion in Theorem 1 equivalent to the inclusion

$$
R(\tau)+R(g) \subseteq P([g, \tau]) .
$$

If $R$ is a division $\operatorname{ring}$, char $R=2, n \geq 2, g=t_{12}(1) t_{21}(1), \tau=t_{12}(1)$, then commutator $[g, \tau]=g^{2}$ commutes with $g$, but does not commute with $\tau$, is not a unipotent element. The inclusion of Theorem 1 does not hold.

Theorem 2. Let $R$ be a division ring, $g \in G L(n, V), n \geq 2, g$ be an arbitrary element, $\tau$ be a transvection such that $\operatorname{dim}(R(\tau)+R(g)) \leq 2$ and the commutator $[g, \tau]$ commutes with $\tau$. Then $[g, \tau]$ commutes with $g$ and there are inclusions

$$
R([g, \tau]) \subseteq P(\tau) \cap P(g), R(\tau)+R(g) \subseteq P([g, \tau]) .
$$

Proof. Theorem 2 is obvious if $g$ and $\tau$ commute. Therefore, in the future we assume that $g$ and $\tau$ do not commute.

The commutator $[g, \tau]$ commutes with the transvection $\tau$ if and only if the transvections $\tau^{g}=g \tau g^{-1}$ and $\tau$ commutes, i.e. when there is a system of inclusions

$$
\left\{\begin{array} { l } 
{ R ( \tau ^ { g } ) \subseteq P ( \tau ) , } \\
{ R ( \tau ) \subseteq P ( \tau ^ { g } ) , }
\end{array} \quad \left\{\begin{array}{l}
g R(\tau) \subseteq P(\tau), \\
R(\tau) \subseteq g P(\tau)
\end{array}\right.\right.
$$

Therefore, the commutator $[g, \tau]$ commutes with the transvection $\tau$ if and only if

$$
g^{ \pm 1} R(\tau) \subseteq P(\tau)
$$

Let $W=R(\tau)+R(g)$. Since $\tau$ is transvection, we have $1 \leq \operatorname{dim} W$. Assume that $\operatorname{dim} W \leq 2$.

If $\operatorname{dim} W=1$, then $R(\tau)=R(g)$ and $g^{ \pm 1} R(\tau)=R(\tau)$. Since $g$ is a unipotent element, $g$ is a transvection, which, contrary to the assumption, commutes with $\tau$.

Let $\operatorname{dim} W=2$. If $R(\tau) \subseteq R(g)$, then $W=R(g)$ and we can assume that $R(\tau)=\left\langle e_{1}\right\rangle$, $R(g)=\left\langle e_{1}, e_{2}\right\rangle$, whee $e_{1}, e_{2}, \ldots, e_{n}$ module base $V$. Therefore, in the base $e_{1}, e_{2}, \ldots, e_{n}$

$$
\tau=\left(\begin{array}{ccc}
1 & r & * \\
0 & 1 & 0 \\
0 & 0 & E
\end{array}\right), \quad g=\left(\begin{array}{ccc}
* & * & * \\
* & * & * \\
0 & 0 & E
\end{array}\right)
$$

If $r \neq 0$, then, up to the conjugation matrix

$$
\left(\begin{array}{lll}
1 & 0 & 0 \\
0 & 1 & * \\
0 & 0 & E
\end{array}\right)
$$

we can assume that $\tau=t_{12}(r)$ i $P(\tau)=\left\langle e_{1}, e_{3}, \ldots, e_{n}\right\rangle$.

The inclusion $g^{ \pm 1} R(\tau) \subseteq P(\tau)$ implies

$$
\tau=\left(\begin{array}{ccc}
1 & r & 0 \\
0 & 1 & 0 \\
0 & 0 & E
\end{array}\right), \quad g=\left(\begin{array}{ccc}
1 & * & * \\
0 & 1 & * \\
0 & 0 & E
\end{array}\right), \quad[g, \tau]=\left(\begin{array}{ccc}
1 & 0 & * \\
& 1 & 0 \\
& & E
\end{array}\right),
$$


Therefore $[g, \tau]$ commutes with $g$ and $R([g, \tau])=\left\langle e_{1}\right\rangle \subseteq P(\tau) \cap P(g)$.

Similarly, if $r=0$, then $P(\tau)$ contains the submodule $\left\langle e_{1}, e_{2}\right\rangle$ and, up to the conjugation,

$$
\tau=\left(\begin{array}{ccc}
1 & 0 & * \\
& 1 & * \\
& & E
\end{array}\right), \quad g=\left(\begin{array}{ccc}
1 & * & * \\
& 1 & * \\
& & E
\end{array}\right), \quad[g, \tau]=\left(\begin{array}{ccc}
1 & 0 & * \\
& 1 & 0 \\
& & E
\end{array}\right) .
$$

Therefore $[g, \tau]$ commutes with $g$ and $R([g, \tau])=\left\langle e_{1}\right\rangle \subseteq P(\tau) \cap P(g)$.

Let $R(\tau)$ do not belong to $R(g)$. Then $R(\tau) \cap R(g)=0, W=R(\tau) \oplus R(g)$. We can assume that $(\tau)=\left\langle e_{1}\right\rangle$.

If $\operatorname{dim} R(g)=1$, then $R(g)=\left\langle e_{2}\right\rangle$. In this case

$$
\tau=\left(\begin{array}{ccc}
1 & r & * \\
0 & 1 & 0 \\
& & E
\end{array}\right), \quad g=\left(\begin{array}{lll}
1 & 0 & 0 \\
l & 1 & * \\
& & E
\end{array}\right)
$$

Since the commutator $[g, \tau]$ commutes with transvection $\tau$, the matrices

commute. So $l r=0$ and

$$
\left(\begin{array}{ll}
1 & 0 \\
l & 1
\end{array}\right)\left(\begin{array}{ll}
0 & r \\
0 & 0
\end{array}\right)\left(\begin{array}{cc}
1 & 0 \\
-l & 1
\end{array}\right) \text { and }\left(\begin{array}{ll}
0 & r \\
0 & 0
\end{array}\right)
$$

if $l=0$ and

$$
[g, \tau]=\left(\begin{array}{ccc}
1 & 0 & * \\
0 & 1 & 0 \\
& & E
\end{array}\right)
$$

if $r=0$.

$$
[g, \tau]=\left(\begin{array}{ccc}
1 & 0 & 0 \\
0 & 1 & * \\
& & E
\end{array}\right)
$$

This means that $R([g, \tau])=\left\langle e_{1}\right\rangle \subseteq P(\tau) \cap P(g)$ if $l=0$ and $R([g, \tau])=\left\langle e_{2}\right\rangle \subseteq$ $P(\tau) \cap P(g)$ if $r=0$. In both cases, the commutator $[g, \tau]$ commutes with $g$ and Theorem 2 is proved.

If $\operatorname{dim}(R(\tau)+R(g)) \geq 3$, then the statement of Theorem 2 does not hold. It is enough to choose $\tau=t_{12}(1), g=t_{24}(1) t_{31}(1)$. Then $g$ is a unipotent element, the commutator $[g, \tau]=t_{14}(-1) t_{32}(1) t_{34}(-1)$ commutes with $\tau$, but the inclusion of Theorem 2 does not hold. Note that the commutator $[g, \tau]$ does not commute with the element $g$ if char $R \neq 2$ and commutes with $g$ if char $R=2$.

Theorem 3. Let $R$ be a division ring, $g \in G L(n, V), n \geq 2, \tau$ be a transvection, so that $[g, \tau]$ is a unipotent element. Then the commutator $[g, \tau, \tau]=[[g, \tau], \tau]$ commutes with $\tau$ and $[g, \tau]$ and there are an inclusions $R([g, \tau, \tau]) \subseteq P(\tau) \cap P([g, \tau]), R(\tau)+R([g, \tau]) \subseteq P[g, \tau, \tau]$.

Proof. Let $\sigma=[g, \tau]$. Then $[g, \tau, \tau]=[\sigma, \tau]$. Under the condition that $\sigma$ is a unipotent element, $R(\sigma) \subseteq R\left(\tau^{g}\right)+R(\tau)=g R(\tau)+R(\tau)$, $\operatorname{dim} R(\sigma) \leq 2$, and $\operatorname{dim}(R(\tau)+R(\sigma)) \leq 2$.

According to Lemma $4 g^{-1} R(\tau) \subseteq P(\tau)$ or $g R(\tau) \subseteq P(\tau)$. By Lemma 1 or Lemma 2, inclusions $R(\tau) \subseteq P(\sigma)$ or $R(\sigma) \subseteq P(\tau)$ are valid.

If $R(\tau) \subseteq P(\sigma)$, then $\sigma^{ \pm 1} R(\tau)=R(\tau) \subseteq P(\tau)$. If $R(\sigma) \subseteq P(\tau)$, then $\left(\sigma^{ \pm 1}-1\right) R(\tau) \subseteq$ $R(\sigma) \subseteq P(\tau)$ and $\sigma^{ \pm 1} R(\tau) \subseteq P(\tau)$. 
In both cases the inclusion $\sigma^{ \pm 1} R(\tau) \subseteq P(\tau)$ holds.

By Lemma 3 , the commutator $[\sigma, \tau]$ commutes with $\tau$.

According to Theorem 2, the commutator $[\sigma, \tau]$ commutes with $\sigma$ and inclusions take place

$$
R([\sigma, \tau]) \subseteq P(\tau) \cap P(\sigma), R(\tau)+R(\sigma) \subseteq P([\sigma, \tau]) .
$$

Theorem 3 can be proved directly without using Theorem 2. After all, without limiting the generality, we can assume that the transvection $\tau$ belongs to the group $t_{1 n}(R)$, and for $g \in G L(n, V), n \geq 2$ there is a Bruhat decomposition $g=s v$, where $s$ is a monomial matrix whose nonzero elements are ones, and $v$ is the upper triangular matrix.

It is easy to see that

$$
s e_{i j}=e_{s(i) j}, e_{i j} s^{-1}=e_{i s(j)}, s e_{i j} s^{-1}=e_{s(i) s(j)}
$$

for any $1 \leq i, j \leq n$. So

$$
[g, \tau] \in t_{1 n}(R)^{s} t_{1 n}(R) \subseteq t_{s(1) s(n)}(R) t_{1 n}(R),[g, \tau, \tau] \subseteq\left[t_{s(1) s(n)}(R), t_{1 n}(R)\right] .
$$

Note that $g_{n 1} \neq 0$ if and only if $s(1)=n$, and $\left(g^{-1}\right)_{n_{1}} \neq 0$ if and only if $s(n)=1$. The commutator $[g, \tau]$ is a unipotent element if and only if $(s(1), s(n)) \neq(n, 1)$, which is equivalent to the equality $g_{n 1}\left(g^{-1}\right)_{n_{1}}=0$.

If the commutator $[g, \tau]$ is a unipotent element, then three cases can occur

1) $[g, \tau]=\left(\begin{array}{ccc}1 & & * \\ & \ddots & \\ & * & 1\end{array}\right), \quad[g, \tau, \tau]=\left(\begin{array}{ccc}1 & * & \\ & \ddots & \\ & & 1\end{array}\right) \in t_{1 s(n)}(R), \quad$ if $\{s(n) \neq 1$,

2) $[g, \tau]=\left(\begin{array}{ccc}1 & & * \\ * & \ddots & * \\ & & 1\end{array}\right), \quad[g, \tau, \tau]=\left(\begin{array}{ccc}1 & & \\ & \ddots & * \\ & & 1\end{array}\right) \in t_{s(1) n}(R), \quad$ if $\left\{\begin{array}{l}s(n)=1 \\ s(1) \neq n\end{array}\right.$

3) $[g, \tau]=\left(\begin{array}{ccc}1 & & * \\ & \ddots & \\ & * & 1\end{array}\right) \in t_{s(1) s(n)}(R) t_{1 n}(R), \quad[g, \tau, \tau]=1, \quad$ if $\left\{\begin{array}{l}s(1) \neq n \\ s(n) \neq 1 .\end{array}\right.$

It is clear that in
1) $\left\{\begin{array}{l}\left(g^{-1}\right)_{n_{1}}=0 \\ g_{n_{1}} \neq 0\end{array}\right.$
2) $\left\{\begin{array}{l}g_{n_{1}}=0 \\ \left(g^{-1}\right)_{n_{1}} \neq 0\end{array}\right.$
3) $g_{n_{1}}=\left(g^{-1}\right)_{n_{1}}=0$.

In all three cases $[g, \tau, \tau]$ commutes with $[g, \tau]$ and $\tau$.

Let $e_{1}, \ldots, e_{n}$ be the basis of the module $V$ such that $R(\tau)=\left\langle e_{1}\right\rangle, P(\tau)=\left\langle e_{1}, \ldots, e_{n-1}\right\rangle$.

Then in cases 1)-3) we have

1) $P([g, \tau]) \in\left\langle e_{1}, \ldots, e_{s(n)-1}, e_{s(n)+1}, \ldots, e_{n-1}\right\rangle, R([g, \tau, \tau]) \in\left\langle e_{1}\right\rangle$,

$$
R([g, \tau]) \in\left\langle e_{1}, e_{n}\right\rangle, P([g, \tau, \tau]) \in\left\langle e_{1}, \ldots, e_{s(n)-1}, e_{s(n)+1}, \ldots, e_{n}\right\rangle .
$$

2) $P([g, \tau]) \in\left\langle e_{2}, \ldots, e_{n-1}\right\rangle, R([g, \tau, \tau]) \in\left\langle e_{s(1)}\right\rangle$,

$$
R([g, \tau]) \in\left\langle e_{1}, e_{s(1)}\right\rangle, P([g, \tau, \tau]) \in\left\langle e_{1}, \ldots, e_{n-1}\right\rangle .
$$

3) $P([g, \tau]) \in\left\langle e_{1}, \ldots, e_{s(n)-1}, e_{s(n)+1}, \ldots, e_{n-1}\right\rangle, R([g, \tau, \tau])=0$,

$$
R([g, \tau]) \in\left\langle e_{1}, e_{s(1)}\right\rangle, P([g, \tau, \tau])=V .
$$


By direct verification we establish that the inclusion of Theorem 3 hold in all three cases.

We emphasize that by the commutators $[g, \tau],[[g, \tau], \tau]$ in Theorem 3 we understand the class of commutators $\left[g, t_{1 n}(r)\right],\left[g, t_{1 n}(r), t_{1 n}\left(r_{1}\right)\right]$ for any elements $r$ and $r_{1}$ of the division ring $R$.

\section{REFERENCES}

1. V.M. Petechuk, Isomorphisms of groups rich in projective transvections, Math. Notes., 39 (1986), №2, 103-107.

2. A.J. Hahn, O.T. O'Meara, The classical groups and K-theory, Berlin, Springer, 1989, 578 p.

3. V.A. Bovdi, V.P. Rudko, Extensions of the representation modules of a prime order group, J. Algebra, 295 (2006), 441-451.

4. N.A. Vavilov, A.V. Stepanov, Linear groups over general rings. I. Generalities, Zap. Nauchn. Sem. S.Petersburg. Otdel. Math. Inst. Steklov. (LOMI), 394 (Voprosy Teorii Predstavlenii Algebr i Grupp. 22), 295 (2011), 33-139.

5. A.E. Zalesskii, Linear groups, Current problems in mathematics. Fundamental directions, 37, Itogi Nauki I Tekhniki, Akad. Nauk SSSR, Vsesoyuz. Inst. Nauchn. I Tekhn. Inform., Moscow, 1989, 114-236. (in Russian)

Transcarpathian Institute of Postgraduate Pedagogical Education

Uzhhorod, Ukraine

vasil.petechuk@gmail.com

Transcarpathian Hungarian Institute named after Ferenc Rakoczi II

Beregovo, Ukraine

yuliia.petechuk @gmail.com

Received 18.02.2020

Revised 19.08.2020 